\title{
Statistical aspects of dissipation by Landau-Zener transitions
}

\author{
Michael Wilkinson $\dagger$ \\ Department of Nuclear Physics, Weizmann Institute of Science, Rehovot, Israel
}

Received 1 March 1988, in final form 31 May 1988

\begin{abstract}
This paper considers the effect of slowly varying the parameters $X_{i}$ of a finite-sized quantum mechanical system. The system is excited to higher energies by Landau-Zener transitions at avoided crossings; since this increases the energy of the system, it has the effect of dissipation of the driving motion. The rate of dissipation depends on the level spacing distribution of the system. When the spectral statistics are those of the Gaussian unitary ensemble, the rate of dissipation is proportional to $\dot{X}_{i}^{2}$, i.e. there is viscous or ohmic damping. When the spectral statistics are of those of the Gaussian orthogonal ensemble, the rate of dissipation is proportional to $\dot{X}_{i}^{3 / 2}$.
\end{abstract}

\section{Introduction}

This paper is concerned with the quantum mechanical description of dissipation in a finite-sized system. The type of system being considered is most clearly stated by giving a specific example. Consider a billiard with a variable shaped boundary, described by some parameters $X_{i}$, and which has chaotic classical motion for all values of $\boldsymbol{X}$ (see figure 1). The billiard has quantum mechanical energy levels $E_{n}(\boldsymbol{X})$, and these are occupied by a large number of non-interacting fermions with probability $f_{n}=f\left(E_{n}\right)$.

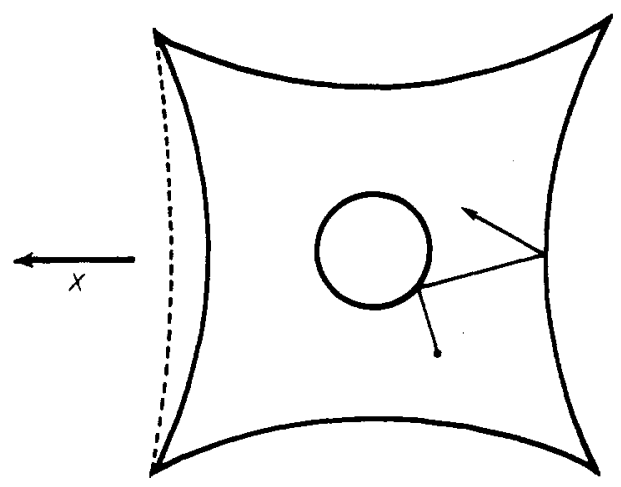

Figure 1. An example of the type of system under consideration. The system is a chaotic billiard with deformable boundaries, described by parameters $X_{1}, X_{2}, \ldots$ The energy levels are occupied by a large number of non-interacting fermions, up to some Fermi level $E_{\mathrm{F}}$. Deforming the boundary produces non-adiabatic excitation of the particles which results in a damping of the driving motion.

$\dagger$ Permanent address: Department of Physics and Applied Physics, John Anderson Building, University of Strathclyde, Glasgow G4 0NG, UK. 
The distribution function $f(E)$ decreases rapidly from unity to zero in the neighbourhood of a 'Fermi energy' $E_{\mathrm{F}}$, but the details of $f(E)$ will not be important in what follows. We imagine that the parameters $X_{i}$ are coupled to some macroscopic system which can be described purely classically. The billiard exerts a force on the macroscopic system. If the rate of change of the parameters, $\dot{X}_{i}$, is small, the dominant contribution to the force is given by the adiabatic approximation:

$$
F_{i}=\operatorname{Tr}\left(\hat{\rho} \frac{\partial \hat{H}}{\partial X_{i}}\right) \approx \sum_{n} f_{n} \frac{\partial E_{n}}{\partial X_{i}}
$$

There are various types of correction to this expression: this paper will discuss the calculation of those corrections which cause damping of the macroscopic motion. The mechanism of this damping is the excitation of the quantum system to higher energy levels.

The principal results of this paper are concerned with the limit where the rate of change $\dot{X}_{i}$ of the parameters is very small. In this case the mechanism of dissipation is by Landau-Zener transitions (this was apparently first suggested as a mechanism for dissipation by Hill and Wheeler (1953) in the context of the collective model for the nucleus). The highly excited energy levels $E_{n}$ exhibit many avoided crossings as a parameter $X_{i}$ is varied (see figure 2). When the gap $\Delta \varepsilon$ in an avoided crossing is sufficiently small, there is a finite probability of a particle making a non-adiabatic transition from an occupied to an unoccupied state. This process was studied by Zener (1932) for the case of an avoided crossing in a two-level system. The difference $\varepsilon$ of the two energy levels as a function of $X$ is parametrised by two constants $\Delta \varepsilon$ and $A$ :

$$
\varepsilon(X)=\left(\Delta \varepsilon^{2}+A^{2} X^{2}\right)^{1 / 2}
$$

(see figure 3 ). Zener showed that the probability of a non-adiabatic transition is

$$
P_{t}=\exp \left(-\frac{\pi \Delta \varepsilon^{2}}{2 A \hbar|\dot{X}|}\right) \text {. }
$$

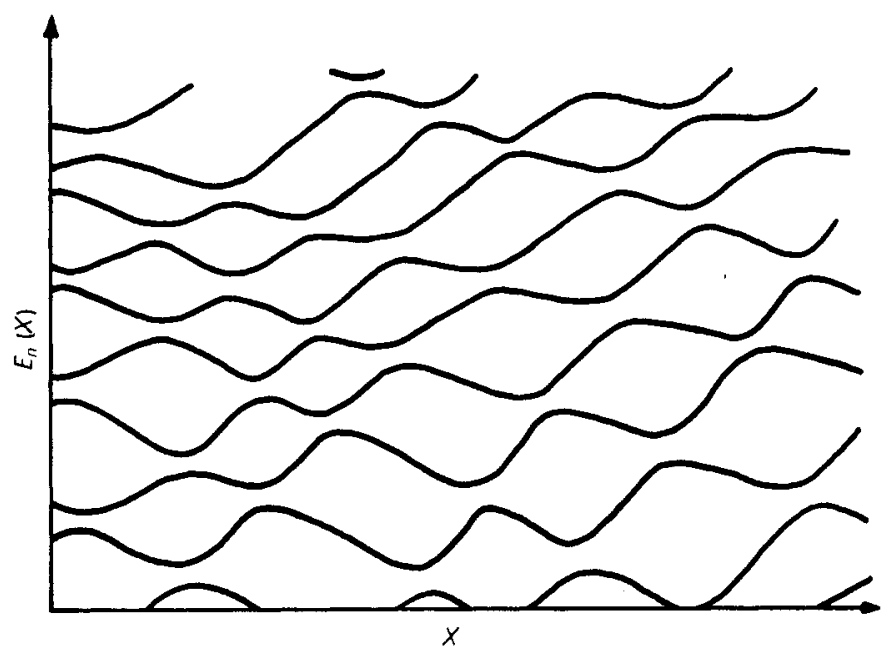

Figure 2. The energy levels of highly excited states, $E_{n}$, exhibit many avoided crossings as a parameter $X_{i}$ is varied. Only those avoided crossings with a sufficiently small gap have significant probability of Landau-Zener transitions. The damping is therefore very sensitive to the level spacing distribution. 


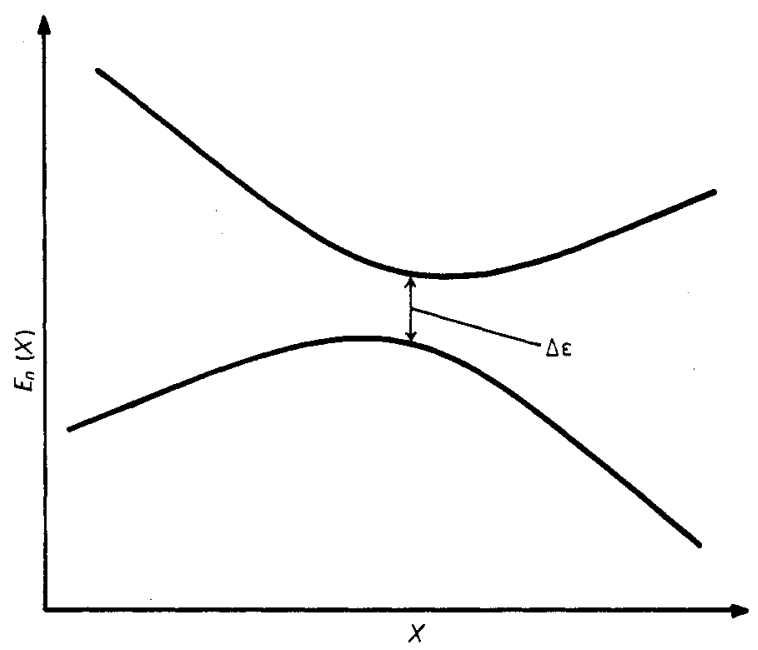

Figure 3. An isolated avoided crossing.

Since the lower-lying energy levels have higher occupation probabilities, these transitions tend to increase the energy of the system, resulting in damping of the driving motion. When the parameter $X$ passes through many avoided crossings, the rate of excitation is determined by the statistical distribution of the gap sizes $\Delta \varepsilon$, which is in turn determined by the level spacing distribution $P(S)$ (i.e. the distribution of the nearest-neighbour separation of energy levels). The dependence of the rate of dissipation on the velocity is calculated in $\S 2$ : it is shown that if

$$
P(S) \propto S^{\nu}
$$

for small $S$, then the rate of dissipation scales as $\dot{X}^{(\nu+2) / 2}$ :

$$
\frac{\partial \bar{E}}{\partial t} \propto n_{0}^{\nu} \hbar^{\nu / 2} \sigma^{(\nu+2) / 2}|\dot{X}|^{(\nu+2) / 2}
$$

where $n_{0}$ is the density of states and $\sigma$ is the typical size of $A$. It is known that the level-spacing distribution, and other statistical properties of the spectrum, show universal behaviour in systems with a chaotic classical limit (Berry 1983, Wilkinson 1988). If the system does not have time-reversal invariance (i.e. if there is a magnetic field present and there are no geometrical symmetries), it is known that the spectral statistics are those of the Gaussian unitary ensemble (GUE) which exhibits quadratic level repulsion ( $\nu=2$ in (1.3)) (Berry and Robnik 1986). In this case the damping force

$$
F_{\mathrm{d}}=\frac{\partial \bar{E}}{\partial t} \frac{1}{\dot{X}}
$$

is proportional to $\dot{X}$, i.e. the damping is viscous. In most other cases the spectral statistics are those of the Gaussian orthogonal ensemble (GOE), which has linear level repulsion $(\nu=1)$. This weaker level repulsion facilitates the Landau-Zener transitions, and causes a larger damping force, proportional to $\dot{X}^{1 / 2}$.

This description of the damping process is only valid when the gap size of those avoided crossings at which there is a significant probability of a transition is much smaller than the mean separation of the energy levels. From (1.3) this condition can 
be written

$$
\hbar \sigma|\dot{X}| n_{0}^{2} \ll 1 .
$$

When $\dot{X}$ is large, so that (1.7) is not satisfied, transitions can occur between states which are not nearest neighbours. In this case it is possible to calculate the rate of dissipation using the Kubo formula (Kubo 1956, Greenwood 1957) and the damping force is proportional to the speed $\dot{X}$, regardless of the nature of the spectral statistics. In the semiclassical limit, the matrix elements appearing in the Kubo formula can be related to a classical correlation function. The relation between the classical correlation function and the dissipative force can also be obtained directly from classical mechanics and the Pauli exclusion principle. These results are described in $\S 3$.

The type of dissipation described here may be relevant to various types of physical problems. The most direct application would be to an extension of the Born-Oppenheimer method in molecular physics, to take account of the damping of the vibrational motion of the nuclei of complex molecules by radiationless transitions of the electronic states. A similar application in nuclear physics would be to the damping of collective coordinates describing deformation of a nucleus by excitation of individual nucleons: this is the context in which Landau-Zener damping was first suggested by Hill and Wheeler (1953). Another possible application of these results is to the electrical conductance of very small samples. Section 4 contains a summary of the important results and some further comments about possible applications.

\section{Dissipation by Landau-Zener transitions}

This section will calculate the rate of increase of the energy of the driven system due to Landau-Zener transitions. For clarity, the calculation is divided into several subsections. Subsection 2.1 calculates the rate of transition $R$ from one level to another, assuming a distribution for the sizes of the gaps of the avoided crossings, $P(\Delta E)$. Subsection 2.2 calculates the relation between $P(\Delta E)$ and the level spacing distribution $P(S)$, so that the transition rate can be calculated. Subsection 2.3 describes some results for estimating the asymptotic slope of the avoided crossings, $A$. Subsection 2.4 relates the transition rate $R$ to the rate of damping $\partial \bar{E} / \partial t$. Subsection 2.5 summarises the important results.

\subsection{Calculation of the transition rate}

The transition rate, $R$, is the probability per unit time that a particle will make a transition to one of the two neighbouring states. To calculate this transition rate we must take account of the fact that the gap sizes $\Delta \varepsilon$ and slopes $A$ of the avoided crossings are randomly distributed. If $N(A, \Delta \varepsilon) \mathrm{d} A \mathrm{~d} \Delta \varepsilon$ is the number of avoided crossings encountered per unit length with slopes in the interval $[A, A+\mathrm{d} A]$ and gap sizes in the interval $[\Delta \varepsilon, \Delta \varepsilon+\mathrm{d} \Delta \varepsilon]$, then in the adiabatic limit we have

$$
R=\dot{X} \int_{0}^{\infty} \mathrm{d} A \int_{0}^{\infty} \mathrm{d} \Delta \varepsilon N(A, \Delta \varepsilon) \exp \left(-\frac{\pi \Delta \varepsilon^{2}}{2 A \hbar \dot{X}}\right)
$$

If random matrix theory is applicable to the system, $N(A, \Delta \varepsilon)$ should be a universal function depending only on the density of states, $n_{0}$, and the typical size of $A$, which will be denoted by $\sigma$. Furthermore, it is expected that $A$ and $\Delta \varepsilon$ should be independent, 
since $A$ is related to the matrix elements of $\partial H / \partial X$, which should have random matrix properties. We can therefore write

$$
N(A, \Delta \varepsilon)=n_{0} A P(\Delta \varepsilon) P^{\prime}(A)
$$

where $P$ and $P^{\prime}$ are universal functions of $n_{0} \Delta \varepsilon$ and $A / \sigma$ respectively, and $P^{\prime}$ is normalised:

$$
\int_{0}^{\infty} \mathrm{d} x P^{\prime}(x)=1
$$

It will be shown in $\$ 2.2$ that the gap sizes have a power law distribution for small $\Delta \varepsilon$

$$
P(\Delta \varepsilon) \mathrm{d} \Delta \varepsilon=\gamma n_{0}^{\nu} \Delta \varepsilon^{\nu-1} \mathrm{~d} \Delta \varepsilon
$$

where $\gamma$ is a dimensionless constant. Combining equations (2.1)-(2.4), we find

$$
R=\gamma I_{\nu} n_{0}^{\nu+1}\left\langle A^{(\nu+2) / 2}\right\rangle \hbar^{\nu / 2}|\dot{X}|^{(\nu+2) / 2}
$$

where the angle brackets denote the average over the distribution of $A$

$$
\left\langle A^{(\nu+2) / 2}\right\rangle=\int_{0}^{\infty} \mathrm{d} A A^{(\nu+2) / 2} P^{\prime}(A)
$$

and

$$
I_{\nu}=\int_{0}^{\infty} \mathrm{d} x x^{\nu-1} \exp \left(-\pi x^{2} / 2\right)
$$

The important cases are $\nu=1,2$, where

$$
I_{1}=2^{-1 / 2} \quad I_{2}=\pi^{-1} .
$$

\subsection{Distribution of gap sizes}

Instead of deriving the distribution of gap sizes (2.3) from the level spacing distribution, $P(S)$, it is easier to carry out the reverse calculation and obtain $P(S)$ from $P(\Delta E)$. For energies much smaller than the mean separation, the level spacing distribution, $P(E)$, is given by

$$
P(E) \mathrm{d} E=\sum 2\left|\frac{\partial \varepsilon}{\partial X}\right|_{\varepsilon(X)=E}^{-1} \mathrm{~d} E
$$

where the sum runs over all the avoided crossings encountered in unit length of the coordinate $X$ with gap separations $\Delta \varepsilon$ smaller than $E$, and $\varepsilon(X)$ is the separation function (1.1) (see figure 3 ). Using (2.2), this summation becomes

$$
P(E) \mathrm{d} E=2 \int_{0}^{\infty} \mathrm{d} A \int_{0}^{E} \mathrm{~d} \Delta \varepsilon N(A, \Delta \varepsilon)\left|\frac{\partial \varepsilon}{\partial X}\right|_{\varepsilon(X)=E}^{-1} \mathrm{~d} E .
$$

Substituting (2.3), the assumed form for $P(\Delta \varepsilon)$, and using the result

$$
\frac{\partial \varepsilon}{\partial X}=\frac{A^{2} X}{\varepsilon}=\frac{A}{\varepsilon}\left(\varepsilon^{2}-\Delta \varepsilon^{2}\right)^{1 / 2}
$$

we find

$P(E) \mathrm{d} E=2 \gamma n_{0}^{\nu+1} \int_{0}^{\infty} \mathrm{d} A A P(A) A^{-1} \int_{0}^{E} \mathrm{~d} \Delta \varepsilon \Delta \varepsilon^{\nu-1} \frac{E}{\left(E^{2}-\Delta \varepsilon^{2}\right)^{1 / 2}} \mathrm{~d} E$. 
Simplifying, we have

$$
P(E) \mathrm{d} E=2 \gamma K_{\nu} n_{0}^{\nu+1} E^{\nu} \mathrm{d} E
$$

where

$$
K_{\nu}=\int_{0}^{1} \mathrm{~d} X \frac{X^{\nu}}{\left(1-X^{2}\right)^{1 / 2}} .
$$

In the important cases, $\nu=1,2$

$$
K_{1}=\pi / 2 \quad K_{2}=1 .
$$

The level spacing distribution normalised to unit mean spacing is therefore

$$
P(S) \mathrm{d} S=2 \gamma K_{\nu} S^{\nu} \mathrm{d} S=\alpha_{\nu} S^{\nu} \mathrm{d} S .
$$

In the case of 'chaotic' systems or disordered systems with extended states, we expect the spectral statistics to be those of the Gaussian orthogonal ensemble (for systems with time-reversal invariance), or the Gaussian unitary ensemble (for systems without time-reversal invariance). These random matrix ensembles and the corresponding spectral statistics are described in a reprint volume edited by Porter (1965). The application of these ensembles to systems with a chaotic classical limit is described by Berry (1983), Bohigas et al (1984), Berry and Robnik (1986) and Wilkinson (1988). The important result in the present context is that when the spectral statistics are of GOE type the level spacing distribution is linear for small $S(\nu=1$ in (2.14)), and for GUE statistics there is quadratic level repulsion $(\nu=2)$. The constants $\alpha_{\nu}$ are as follows:

$$
\begin{array}{lll}
\text { GOE } & \nu=1 & \alpha_{1}=\pi^{2} / 6 \\
\text { GUE } & \nu=2 & \alpha_{2}=\pi^{2} / 3
\end{array}
$$

(Dyson 1962). Using these results, we can calculate the dimensionless factor $\gamma$ appearing in (2.4).

\subsection{Distribution of slopes of avoided crossings}

The slope $A$ of an avoided crossing is clearly related to the matrix elements of the operator $(\partial \hat{H} / \partial X)$. This operator bears no relation to the eigenstates of the Hamiltonian $\hat{H}(\boldsymbol{X})$, and the matrix elements $(\partial \hat{H} / \partial X)$ should satisfy the statistical hypotheses of random matrix theory (Porter 1965). We therefore expect that these matrix elements are independently Gaussian distributed, with a variance $\sigma^{2}$ which varies slowly with $E=\frac{1}{2}\left(E_{n}+E_{m}\right)$, and $\Delta E=E_{n}-E_{m}$ :

$\left\langle\left|\left(\frac{\partial \hat{H}}{\partial X}\right)_{n m}\right|^{2}\right\rangle=\sigma^{2}(E, \Delta E)=\frac{1}{n_{0}^{2}} \sum_{n m}\left|\left(\frac{\partial \hat{H}}{\partial X}\right)_{n m}\right|^{2} \delta_{\varepsilon}\left(E-\frac{1}{2}\left(E_{n}+E_{m}\right)\right) \delta_{\varepsilon}\left(\Delta E-\left(E_{n}-E_{m}\right)\right)$

where $\delta_{\varepsilon}(x)$ represents a Dirac $\delta$ function smeared out over a suitably chosen range of width $\varepsilon$. In the GOE case variance of the diagonal matrix elements is different from that of the nearby off-diagonal matrix elements:

$$
\left\langle\left(\frac{\partial \hat{H}}{\partial X}\right)_{n n}^{2}\right\rangle=\beta_{\nu}\left\langle\left|\left(\frac{\partial \hat{H}}{\partial X}\right)_{n m}\right|^{2}\right\rangle_{E_{n}=E_{m}}=\beta_{\nu} \sigma^{2}(E, 0)
$$


where

$$
\begin{array}{ll}
\text { GOE } & \beta_{1}=2 \\
\text { GUE } & \beta_{2}=1 .
\end{array}
$$

The scale size of the slopes is characterised by the variance $\sigma^{2}$ of the matrix elements of $(\partial \hat{H} / \partial X)$, but the probability distribution $P^{\prime}(A)$ is not Gaussian. In appendix 1 it is shown that, in the GOE case,

$$
\text { GOE } \quad P^{\prime}(A) \mathrm{d} A=\frac{A}{4 \sigma^{2}} \exp \left(-\frac{A^{2}}{8 \sigma^{2}}\right) \mathrm{d} A
$$

and in the GUE case

$$
\text { GUe } \quad P^{\prime}(A) \mathrm{d} A=\frac{A^{2}}{2 \sqrt{\pi} \sigma^{3}} \exp \left(-\frac{A^{2}}{4 \sigma^{2}}\right) \mathrm{d} A .
$$

We can now evaluate the moments of $A$ appearing in (2.5). The results are

$$
\text { GOE } \quad\left\langle A^{3 / 2}\right\rangle=\frac{1}{4 \sigma^{2}} \int_{0}^{\infty} \mathrm{d} A A^{5 / 2} \exp \left(\frac{A^{2}}{8 \sigma^{2}}\right)=3.2^{1 / 4} \Gamma\left(\frac{3}{4}\right) \sigma^{3 / 2}
$$

where the value of the gamma function is $\Gamma\left(\frac{3}{4}\right)=1.22541$, and

$$
\text { GUE }\left\langle A^{2}\right\rangle=\frac{1}{2 \sqrt{\pi} \sigma^{3}} \int_{0}^{\infty} \mathrm{d} A A^{4} \exp \left(-\frac{A^{2}}{4 \sigma^{2}}\right)=6 \sigma^{2} .
$$

Finally, a result will be described which enables the variance $\sigma^{2}$ to be calculated from classical quantities in systems which have a chaotic classical limit. In these cases the density of states is given by the Weyl formula (Berry 1983)

$$
n_{0}=\frac{\Omega}{(2 \pi \hbar)^{d}}
$$

where $d$ is the number of degrees of freedom, and

$$
\Omega=\int \mathrm{d}^{d} p \mathrm{~d}^{d} q \delta(E-H(\boldsymbol{p}, \boldsymbol{q})) .
$$

The variance of the matrix elements of an operator $\hat{A}$ which has a classical limit $\boldsymbol{A}(\boldsymbol{p}, \boldsymbol{q})$ can be written in terms of the classical correlation function of $A(p, q)$ :

$$
\sigma^{2}(E, 0)=(2 \pi \hbar)^{d-1} \Omega^{-2} \int_{-\infty}^{\infty} \mathrm{d} t C_{A}(E, t)
$$

where the correlation function is given by

$$
C_{A}(E, t)=\int \mathrm{d}^{d} p \mathrm{~d}^{d} q A(p, q) A(p(t), q(t)) \delta(E-H(p, q))
$$

(Wilkinson 1987). Using equations (2.26)-(2.29) it is possible to express the transition rate in terms of classical quantities and $\hbar$ only, provided the integral in (2.28) converges. 


\subsection{Rate of dissipation}

The $n$th state of the system is filled with a probability $f_{n}$. The rate of change of $f_{n}$ is given by

$$
\mathrm{d} f_{n} / \mathrm{d} t=\left(-2 f_{n}+f_{n+1}+f_{n-1}\right) R .
$$

This result assumes a random phase approximation which is discussed in appendix 2 . This approximation is probably justified in the limit where $\dot{X}$ is small because the avoided crossings which have a significant transition probability are widely separated. If the probabilities $f_{n}$ vary slowly with the level number $n$, we can write

$$
f_{n}(t)=f\left(E_{n}, t\right)
$$

and (2.30) shows that $f(E, t)$ satisfies a diffusion equation

$$
\frac{\partial f}{\partial t}=\frac{R}{n_{0}^{2}} \frac{\partial^{2} f}{\partial E^{2}} .
$$

The total energy of the system is given by

$$
\bar{E}(t)=\int \mathrm{d} E n_{0}(E) f(E, t) E
$$

or, if we consider the case in which the Fermi level is sharply defined,

$$
\bar{E}(t)=n_{0}\left(E_{\mathrm{F}}\right) \int \mathrm{d} E E f(E, t) .
$$

The rate of increase of the energy of the system due to Zener transitions is

$$
\frac{\partial \bar{E}}{\partial t}=n_{0} \int \mathrm{d} E E \frac{\partial f}{\partial t}=\frac{R}{n_{0}} \int \mathrm{d} E E \frac{\partial^{2} f}{\partial E^{2}}=-\frac{R}{n_{0}} \int \mathrm{d} E \frac{\partial f}{\partial E}
$$

so that

$$
\frac{\partial \bar{E}}{\partial t}=\frac{R}{n_{0}}
$$

This energy is supplied by the driving motion, and in most cases it is not recoverable, so that it represents dissipation of the driving motion. The damping force is given by

$$
F_{\mathrm{d}}=\frac{\partial \bar{E}}{\partial X}=\frac{R}{n_{0} \dot{X}} .
$$

This force always opposes the direction of the motion.

\subsection{Summary}

In the adiabatic limit (1.7) the rate of dissipation is very sensitive to the level spacing distribution. In systems with GOE spectral statistics, which have linear level repulsion, our estimate of the rate of dissipation is (combining $(2.4),(2.14),(2.15)$ and (2.24))

$$
\text { GOE } \quad \frac{\partial \bar{E}}{\partial t}=2^{-5 / 4} \pi \Gamma\left(\frac{3}{4}\right) n_{0} \hbar^{1 / 2}\left[\sigma^{2}\left(E_{\mathrm{F}}, 0\right)\right]^{3 / 4}|\dot{X}|^{3 / 2}
$$


where $\sigma^{2}(E, 0)$ is the variance of the matrix elements of $\partial \hat{H} / \partial X$. If the system has GUE spectral statistics, the estimate of the rate of dissipation is

$$
\text { GUE } \quad \frac{\partial \bar{E}}{\partial t}=\pi \hbar n_{0}^{2} \sigma^{2}(E, 0) \dot{X}^{2} .
$$

From (2.37) we see that in this case the damping force is proportional to $\dot{X}$, corresponding to ohmic or viscous damping, whereas in the GOE case the damping force is larger and is proportional to $\dot{X}^{1 / 2}$.

If the system has a chaotic classical limit, with correlation function decaying faster than $1 / t$, then equations $(2.26)-(2.29)$ can be used to express $\sigma^{2}$ in terms of the correlation function of the classical motion.

\section{Rate of dissipation at higher velocities}

The results of $\S 2$ assume that the velocity at which the system is driven is very slow, so that (1.7) is satisfied. When the velocity exceeds this limit, the rate of dissipation can be calculated from the Kubo formula: $\S 3.1$ gives a brief discussion of this formula and the conditions under which it is applicable, which are the opposite of (1.7).

In systems with a classical limit, the Kubo formula should give the correct rate of dissipation in the semiclassical limit, $\hbar \rightarrow 0$, since the density of states, $n_{0}$, scales as $\hbar^{-d}$, so that (1.7) is not satisfied. It should therefore be possible to derive the rate of dissipation in this limit directly from classical arguments, together with the Pauli exclusion principle. This is described in $\$ 3.2$.

\subsection{The Kubo formula}

There are many related formulae for electrical conductivity and other linear response properties which are known as Kubo formulae (Kubo 1956, Greenwood 1957, Mahan 1980). The form most relevant to the results of this paper is that described by Greenwood (1957) and the discussion below follows the method described in that paper. The problem is to calculate a generalised force defined by

$$
\langle F(t)\rangle=\operatorname{Tr}\left(\hat{\rho}(t) \frac{\partial \hat{H}}{\partial X}\right) .
$$

In most discussions this force is an electric current and the conjugate coordinate is the vector potential. The density matrix is assumed to have a part which is diagonal in the instantaneous eigenstates $|n(t)\rangle=|n(X(t))\rangle$, and a perturbation term which is assumed to be proportional to $\dot{X}$ :

$$
\hat{\rho}(t)=\sum_{n}|n(t)\rangle f_{n}\langle n(t)|+\hat{\rho}_{1} \dot{X}
$$

The force is then given by

$$
\langle F(t)\rangle=\left.\sum_{n} f_{n} \frac{\partial E_{n}}{\partial X}\right|_{X(t)}+\mu \dot{X}
$$

where the first term is the usual adiabatic approximation (1.1) and the coefficient $\mu$ represents a viscosity, or a conductivity in the electrical context. Following the method of calculation described by Greenwood, we substitute (3.2) into the equation of motion 
for the density matrix, solve for $\hat{\rho}_{1}$, and substitute into (3.7). The resulting expression for the viscosity is

$$
\mu=\pi \hbar \sum_{\substack{n, m \\ n \neq m}}\left|\left(\frac{\partial H}{\partial X}\right)_{m m}\right|^{2}\left(\frac{\partial f}{\partial E}\right)_{E_{n}} \delta_{\varepsilon}\left(E_{n}-E_{m}\right) .
$$

Here $\delta_{\varepsilon}$ is a delta function broadened over a width $\varepsilon=\hbar / \tau$, where $\tau$ is the length of time over which the perturbation acts. The method of solution assumes that the energies $E_{n}, E_{m}$ are constant, whereas in fact they depend on $X(t)$. This assumption is justified if $E_{n}-E_{m}$ is much larger than the mean level spacing for most of the pairs of levels which contribute to the sum, i.e.

$$
\varepsilon n_{0}=\tau^{-1} \hbar n_{0} \gg 1 \text {. }
$$

If the occupation probability $f_{n}$ drops sharply at the Fermi level $E_{\mathrm{F}}$, then we can write

$$
\left.\frac{\partial f}{\partial E}\right|_{E_{n}} \simeq \delta_{\varepsilon}\left(E_{n}-E_{\mathrm{F}}\right)
$$

so that the viscosity is

$$
\mu=\pi \hbar n_{0}^{2} \sigma^{2}\left(E_{\mathrm{F}}, 0\right)
$$

where $\sigma^{2}$ is the variance of the matrix elements of $\partial \hat{H} / \partial X$, defined by (2.18). When the system considered has a classical limit, the viscosity can be related to the classical correlation function, (2.28) and (2.29):

$$
\mu=\frac{n_{0}}{2 \Omega} \int_{-\infty}^{\infty} \mathrm{d} t C\left(E_{\mathrm{F}}, t\right) .
$$

The corresponding formula for the rate of dissipation is

$$
\partial \bar{E} / \partial t=\pi \hbar n_{0}^{2} \sigma^{2} \dot{X}^{2} .
$$

Now compare this result with those obtained in $\S 2$. The results on Landau-Zener transitions assumed that the energy levels make many avoided crossings as the parameter $X$ varies; the condition for this can be written

$$
\dot{X} \tau \sigma n_{0} \gg 1
$$

where $\tau$ is the timescale over which the motion occurs. If there are many avoided crossings, the condition (3.5) for the Kubo formula to be valid can therefore be written

$$
n_{0}^{2} \dot{X} \sigma \hbar \gg 1
$$

which is exactly the opposite of the condition for the Landau-Zener effect to be the mechanism of dissipation (1.7). There is a crossover between the two mechanisms when $\sigma \hbar \dot{X} n_{0}^{2} \simeq 1$, when the rate of dissipation predicted by the two approaches has the same order of magnitude (compare (3.9) with (2.38) and (2.39)). The fact that the dimensionless prefactor is the same in (2.39) and (3.9) appears to be a coincidence.

\subsection{Semiclassical calculation of the dissipation rate}

If the system has a classical limit, it should be possible to relate the rate of dissipation directly to properties of the classical dynamics. Many of the earlier results relied on the quantum system having random matrix properties, which are associated with 
systems having a chaotic classical limit. For this reason it will be assumed that the classical dynamics is chaotic.

First consider the semiclassical limit of the conservative force, (1.1). The derivatives of the energy levels $E_{n}$ with respect to $X$ can be deduced from the fact that ergodic systems have the following adiabatic invariant (Ott 1979):

$$
I=\int \mathrm{d}^{d} q \mathrm{~d}^{d} p \theta(E-H(q, p, X(t)))
$$

where $\theta$ is the step function. This invariant implies that the volume of the energy shell remains constant for slow changes of the Hamiltonian. The rate of change of energy with the parameter $X$ is therefore given by

$$
\frac{\partial E}{\partial X}=\left\langle\frac{\partial H}{\partial X}\right\rangle=\int \mathrm{d}^{d} q \mathrm{~d}^{d} p \delta(E-H(q, p, X(t))) \frac{\partial H}{\partial X}(q, p, X(t)) .
$$

If the parameter $X(t)$ is varied sufficiently slowly, the system will remain in a state with the same quantum number, so that $\partial E_{n} / \partial X \simeq \partial E / \partial X$. The semiclassical limit of the conservative force $(1.1)$ is therefore given by

$$
F=\int^{E_{\mathrm{F}}} \mathrm{d} E n_{0}(E) \frac{\partial E}{\partial X}
$$

where $\partial E / \partial X$ is given by (3.13).

The dissipative contribution to the force comes from the fact that there is a diffusive spread of the energy of the particles about the mean value obtained by integrating (3.13) (Ott 1979). The rate of change of energy of a particle is given by

$$
\frac{\mathrm{d} E}{\mathrm{~d} t}=\frac{\partial H}{\partial t}=\frac{\partial H}{\partial X} \dot{X}
$$

so that the difference between the change in energy given by (3.13) and the exact value is

$$
\Delta E(\boldsymbol{q}, \boldsymbol{p}, T)=\int_{0}^{T} \mathrm{~d} t \dot{X}\left(\frac{\partial H}{\partial X}(q(t), p(t), X(t))-\left\langle\frac{\partial H}{\partial X}\right\rangle\right)
$$

for a particle starting at $(\boldsymbol{q}, \boldsymbol{p})$. The ergodic theorem implies that

$$
\lim _{T \rightarrow \infty} \frac{\Delta E}{T} \rightarrow 0
$$

which justifies the assertion that (3.12) is an adiabatic invariant. The variance of $\Delta E$ (averaged over the initial position $(\boldsymbol{q}, \boldsymbol{p})$ ) is given by

$$
\begin{aligned}
\left\langle\Delta E\left(T^{2}\right)\right\rangle=\frac{1}{\Omega} & \int \mathrm{d}^{d} q \mathrm{~d}^{d} p \delta(E-H(\boldsymbol{q}, \boldsymbol{p})) \int_{0}^{T} \mathrm{~d} t_{1} \int_{0}^{T} \mathrm{~d} t_{2} \dot{X}\left(t_{1}\right) \dot{X}\left(t_{2}\right) \\
& \times\left(\frac{\partial H}{\partial X}\left(t_{1}\right)-\left\langle\frac{\partial H}{\partial X}\right\rangle\right)\left(\frac{\partial H}{\partial X}\left(t_{2}\right)-\left\langle\frac{\partial H}{\partial X}\right\rangle\right)
\end{aligned}
$$

where $\Omega$ is defined by (2.27). Exchanging the order of the integrals over time and phase space in (3.18), and assuming that $T$ is much greater than the timescale for the 
decay of correlations, we have

$$
\left\langle\Delta E(T)^{2}\right\rangle=\int_{0}^{T} \mathrm{~d} t \frac{\dot{X}^{2}}{\Omega} \int_{-\infty}^{\infty} \mathrm{d} \tau C(E, \tau)
$$

where $\tau=t_{1}-t_{2}$ and $C(E, \tau)$ is the correlation function of $\partial H / \partial X$, defined by (2.29). The energy of a particle therefore diffuses about the mean value given by (3.13), with diffusion constant

$$
D=\frac{\dot{X}^{2}}{2 \Omega} \int_{-\infty}^{\infty} \mathrm{d} \tau C(E, \tau)
$$

From (2.32) and (2.36) this implies that the rate of dissipation is given by

$$
\frac{\partial \bar{E}}{\partial t}=\mu \dot{X}^{2}=\frac{n_{0} \dot{X}^{2}}{2 \Omega} \int_{-\infty}^{\infty} \mathrm{d} \tau C(E, \tau)
$$

where the viscosity $\mu$ is the same as that obtained using the Kubo formula (3.8).

\section{Summary and concluding remarks}

This paper has considered the problem of dissipation in a finite-sized quantum system, caused by slowly varying a set of external parameters $X_{i}$. When the velocity is small, the mechanism of dissipation is by excitation of the system by Landau-Zener transitions. This mechanism is very sensitive to the form of the level spacing distribution: if the level spacing distribution has a power law with exponent $\nu(1.4)$, then the rate of dissipation scales as indicated in (1.5). Some plausible assumptions were used to estimate the dimensionless prefactor of (1.5) in the cases of GOE statistics $(\nu=1)$ and GUE statistics $(\nu=2)$ : these results are summarised in $\S 2.5$.

These results are only valid when $|\dot{X}|$ is sufficiently small: when $n_{0}^{2} \sigma \hbar|\dot{X}| \simeq 1$ Landau-Zener transitions cease to be the dominant mechanism of dissipation, and the rate of dissipation crosses over to a value given by the Kubo formula which is independent of the spectral statistics (see figure 4).

As well as the applications to molecular and nuclear physics described in the introduction, it may be possible to devise systems where these results could be tested experimentally. The theory described in $\$ 2$ makes two predictions which could be tested without a detailed microscopic knowledge of the system. First, the dissipative force is proportional to $|\dot{X}|^{1 / 2}$ for the case of GOE statistics, a result which would be hard to explain in any other way. Secondly, if a magnetic field is applied which is sufficiently strong to alter the spectral statistics from GOE to GUE, the dissipative force is greatly reduced, and is proportional to $|\dot{X}|$. The condition for this is that the field should be large enough to shift energy levels by an amount comparable to their spacing. Perhaps the absorption of electromagnetic radiation by very small metallic particles could provide a suitable system on which to test these predictions.

Another possible application is to conduction in small metallic rings through which a magnetic flux $\phi$ is threaded. The magnetic flux is analogous to the coordinate $X$, 


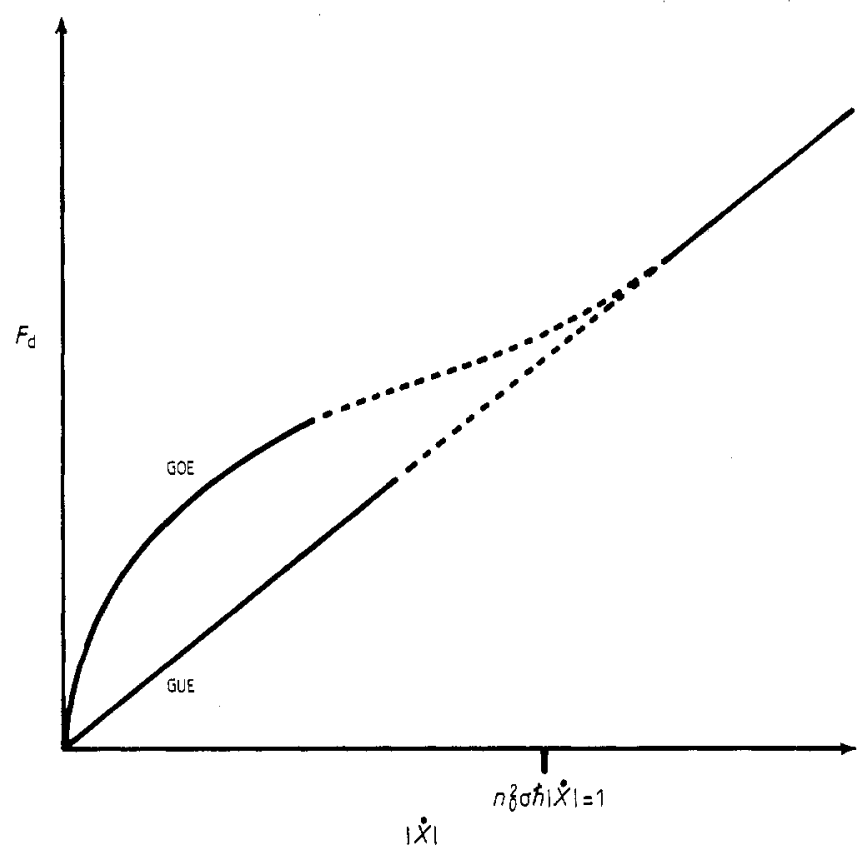

Figure 4. When $|\dot{X}|$ is small, the damping force depends on the spectral statistics: for GOE statistics it is non-linear, proportional to $|\dot{X}|^{1 / 2}$, and for GUE statistics it is linear (proportional to $|\dot{X}|$ ). At larger values of $|\dot{X}|$ there is a crossover to a linear relationship given by the Kubo formula.

and the velocity $\dot{X}$ corresponds to an EMF induced in the ring:

$$
V=\dot{\phi}
$$

The analogue of the force $\partial H / \partial X$ is the electric current:

$$
J=\operatorname{Tr}\left(\hat{\rho} \frac{\partial \hat{H}}{\partial \phi}\right) .
$$

The eigenvalues $E_{n}(\phi)$ are periodic in $\phi$ with period $e / h$ (one flux quantum). Systems of this type have been discussed in several earlier papers (e.g. Büttiker et al 1983, Gefen and Thouless 1987); usually it is assumed that the wire is effectively one dimensional, which implies that $E_{n}(\phi)$ has only one maximum and minimum per period. In the (possibly more realistic) situation in which the ring is not one dimensional, there will be many avoided crossings per period, and the results of this paper may be applicable. The nature of the dissipation would then depend on the spectral statistics. The eigenvalues of an essentially similar type of system (a chaotic billiard with a magnetic flux $\phi$ concentrated at a point within the boundary) have been investigated by Berry and Robnik (1986) and their results are also expected to apply to the conducting loop. The spectral statistics are those of the Gaussian orthogonal ensemble (GOE) when $\phi$ is an integer or half-integer number of flux quanta, but quickly transform to unitary (GUE) statistics when $\phi$ is shifted away from these values. The current would apparently therefore satisfy Ohm's law even when LandauZener transitions are the mechanism for dissipation. 


\section{Acknowledgments}

I am grateful to the Weizmann Institute for their hospitality and to the Royal Society for funding my visit there. I also benefited from useful discussions with Professor $M$ $\mathrm{V}$ Berry and Professor Y Gefen.

\section{Appendix 1}

This appendix will calculate the dependence of the density of avoided crossings, $N(A, \Delta \varepsilon)$, on the slope parameter $A$, in the limit where the gap $\Delta \varepsilon$ is small (only in this limit is the asymptotic slope $A$ well defined).

The method is as follows. We assume that the energy levels $E_{n}$ and the matrix elements of the operator $(\partial \hat{H} / \partial X)$ have been evaluated at $X=X_{0}$. Using these values, we can calculate the position of the nearest avoided crossing to $X_{0}$ for pairs of energy levels which are very close together (for which degenerate perturbation theory can be applied). The density $N(A, \Delta \varepsilon)$ is the probability that an avoided crossing of slope $A$ and gap $\Delta \varepsilon$ lies between $X_{0}$ and $X_{0}+\mathrm{d} X$, divided by the increment $\mathrm{d} X$ : if $\lambda$ is the displacement of the position of the avoided crossing from $X_{0}$, then

$$
\begin{aligned}
& N\left(A^{\prime}, \Delta \varepsilon^{\prime}\right) \mathrm{d} A^{\prime} \mathrm{d} \Delta \varepsilon^{\prime} \\
& \quad=\lim _{\mathrm{d} X \rightarrow 0} P\left[0<\lambda<\mathrm{d} X, \Delta \varepsilon^{\prime}<\Delta \varepsilon<\Delta \varepsilon^{\prime}+\mathrm{d} \Delta \varepsilon^{\prime}, A^{\prime}<A<A^{\prime}+\mathrm{d} A^{\prime}\right] .
\end{aligned}
$$

In order to calculate the probability appearing on the RHS of (A1.1) it is necessary to know how the parameters $A, \Delta \varepsilon, \lambda$ describing the avoided crossing are related to the eigenvalues $E_{n}$ and matrix elements $(\partial \hat{H} / \partial X)_{n m}$, and also the probability distribution of these latter quantities. Consider first the relationship between the parameters of the avoided crossing, and the energy levels and matrix elements. Assume that the eigenvalues $E_{n}$ and $E_{n+1}$ are very nearly degenerate at $X_{0}$. In the neighbourhood of $X_{0}$ the behaviour of these two energy levels can be described by degenerate perturbation theory: the energy levels are eigenvalues of the $2 \times 2$ matrix

$$
\hat{H}=\left[\begin{array}{ll}
e^{+} & h \\
h^{*} & e^{-}
\end{array}\right]
$$

the matrix elements of which vary linearly in $X$ :

$$
\begin{aligned}
& e^{-}=e_{0}^{-}+e_{1}^{-}\left(X-X_{0}\right)=E_{n}+\left(\frac{\partial \hat{H}}{\partial X}\right)_{n n}\left(X-X_{0}\right) \\
& e^{+}=e_{0}^{+}+e_{1}^{+}\left(X-X_{0}\right)=E_{n}+\left(\frac{\partial \hat{H}}{\partial X}\right)_{n+1, n+1}\left(X-X_{0}\right) \\
& h=h_{1}\left(X-X_{0}\right)=\left(\frac{\partial \hat{H}}{\partial X}\right)_{n, n+1} \quad\left(X-X_{0}\right)
\end{aligned}
$$

where the energy levels and matrix elements on the RHS of (A1.3)-(A1.5) are evaluated at $X=X_{0}$. The difference between the two eigenvalues of (A1.2) is given by

$$
\Delta \varepsilon(X)=\left(e^{2}+4|h|^{2}\right)^{1 / 2}
$$

where

$$
e=e^{+}-e^{-}=e_{0}+e_{1}\left(X-X_{0}\right)
$$


Equation (A1.6) should be compared with the equation defining the parameters of the avoided crossing

$$
\Delta \varepsilon(X)=\left[\Delta \varepsilon^{2}+A^{2}\left(X-X_{0}-\lambda\right)^{2}\right]^{1 / 2} .
$$

Substituting (A1.3)-(A1.5) into (A1.6), and comparing with (A1.8), we find the following expressions for the parameters of the avoided crossing:

$$
\begin{aligned}
& A=\left(e_{1}^{2}+4\left|h_{1}\right|^{2}\right)^{1 / 2} \\
& \lambda=e_{0} e_{1} / A^{2}=e_{0} e_{1} /\left(e_{1}^{2}+4\left|h_{1}\right|^{2}\right) \\
& \Delta \varepsilon=\left(e_{0}^{2}-A^{2} \lambda^{2}\right)^{1 / 2}=2\left|h_{1}\right| e_{0} /\left(e_{1}^{2}+4\left|h_{1}\right|^{2}\right)^{1 / 2} .
\end{aligned}
$$

Next consider the probability distribution of the quantities $e_{0}, e_{1}, h_{1}$ appearing in (A1.9)-(A1.11). If random matrix theory is applicable to the Hamiltonian $H(X)$, then the matrix elements of $(\partial \hat{H} / \partial X)$ are independently Gaussian distributed, as described in $\$ 2.3$. In the GOE case, we see from (2.19)-(2.21) and (A1.3)-(A1.7) that $e_{1}$ and $h_{1}$ are Gaussian distributed with variances $4 \sigma^{2}$ and $\sigma^{2}$ respectively:

$$
\begin{aligned}
& P\left(e_{1}\right) \mathrm{d} e_{1}=\frac{1}{2 \sqrt{2 \pi} \sigma} \exp \left(-\frac{e_{1}^{2}}{8 \sigma^{2}}\right) \\
& P\left(h_{1}\right) \mathrm{d} h_{1}=\frac{1}{\sqrt{2 \pi} \sigma} \exp \left(-\frac{h_{1}^{2}}{2 \sigma^{2}}\right) \mathrm{d} h_{1} .
\end{aligned}
$$

In the GUE case, the off-diagonal matrix element $h$ is complex, and the real and imaginary parts of $h_{1}$ are independently Gaussian distributed, each with variance $\frac{1}{2} \sigma^{2}$. The probability distribution of $e_{0}$ is just the level spacing distribution, given by (2.16)-(2.18).

Given (A1.9)-(A1.11) and the probability distributions for $e_{0}, e_{1}$ and $h_{1}$ the RHS of (A1.1) can now be evaluated. In the GOE case

$N\left(A^{\prime}, \Delta \varepsilon^{\prime}\right) \mathrm{d} A^{\prime} \mathrm{d} \Delta \varepsilon^{\prime}$

$$
\begin{aligned}
= & \lim _{\Delta X \rightarrow 0} \frac{1}{\Delta X} \int \mathrm{d} e_{0} \int \mathrm{d} e_{1} \int \mathrm{d} h_{1} P\left(e_{0}\right) P\left(e_{1}\right) P\left(h_{1}\right) \delta\left(A^{\prime}-A\left(e_{0}, e_{1}, h_{1}\right)\right) \\
& \times \delta\left(\Delta \varepsilon^{\prime}-\Delta \varepsilon\left(e_{0}, e_{1}, h_{1}\right)\right) \\
& \times \theta\left(\lambda\left(e_{0}, e_{1}, h_{1}\right)\right) \theta\left(\Delta X-\lambda\left(e_{0}, e_{1}, h_{1}\right)\right) \mathrm{d} A^{\prime} \mathrm{d} \Delta \varepsilon^{\prime}
\end{aligned}
$$

where $\theta(x)$ is the unit step function. Taking the limit $\Delta X \rightarrow 0$

$N\left(A^{\prime}, \Delta \varepsilon^{\prime}\right) \mathrm{d} A^{\prime} \mathrm{d} \Delta \varepsilon^{\prime}$

$$
\begin{aligned}
= & \int \mathrm{d} e_{0} \int \mathrm{d} e_{1} \int \mathrm{d} h_{1} P\left(e_{0}\right) P\left(e_{1}\right) P\left(h_{1}\right) \delta\left(A^{\prime}-A\right) \\
& \times \delta\left(\Delta \varepsilon^{\prime}-\Delta \varepsilon\right) \delta(\lambda) \mathrm{d} A^{\prime} \mathrm{d} \Delta \varepsilon^{\prime}
\end{aligned}
$$

where $A, \Delta \varepsilon, \lambda$ are functions of $e_{0}, e_{1}, h_{1}$ given by (A1.9)-(A1.11). To calculate the $A^{\prime}$ dependence of $N\left(A^{\prime}, \Delta \varepsilon^{\prime}\right)$, it will be useful to change the variables $e_{1}, h_{1}$ to polar coordinates $r, \theta$ :

$$
e_{1}=r \cos \theta \quad h_{1}=\frac{1}{2} r \sin \theta .
$$


In the new variables $(\mathrm{A} 1.15)$ becomes

$$
\begin{aligned}
N\left(A^{\prime}, \Delta \varepsilon^{\prime}\right)= & \text { constant } \times \int \mathrm{d} e_{0} P\left(e_{0}\right) \int \mathrm{d} \theta \int \mathrm{d} r r \exp \left(-\frac{r^{2}}{8 \sigma^{2}}\right) \delta\left(r-A^{\prime}\right) \\
& \times \delta\left(\Delta \varepsilon^{\prime}-e_{0} \sin \theta\right) \delta\left(e_{0} \cos \theta / r\right) .
\end{aligned}
$$

Using the result

$$
\delta\left(e_{0} \cos \theta / r\right)=\frac{r}{e_{0}} \delta\left(\left(\theta-\frac{1}{2} \pi\right) \bmod \pi\right)
$$

(A1.17) becomes

$$
\begin{gathered}
N\left(A^{\prime}, \Delta \varepsilon^{\prime}\right)=\text { constant } \times \int \mathrm{d} e_{0} P\left(e_{0}\right) e_{0}^{-1} \delta\left(\Delta \varepsilon^{\prime}-e_{0}\right) \\
\times \int \mathrm{d} r r^{2} \exp \left(-\frac{r^{2}}{8 \sigma^{2}}\right) \delta\left(A^{\prime}-r\right) .
\end{gathered}
$$

Thus in the GOE case

$$
N(A, \Delta \varepsilon)=\text { constant } \times A^{2} \exp \left(-\frac{A^{2}}{8 \sigma^{2}}\right)
$$

since $P\left(e_{0}\right)$ is proportional to $e_{0}$. Comparing this result with equation (2.2), we see that the normalised distribution $P^{\prime}(A)$ is given by

$$
P^{\prime}(A)=\frac{A}{4 \sigma^{2}} \exp \left(-\frac{A^{2}}{8 \sigma^{2}}\right)
$$

as claimed in $\$ 2.3$

A similar calculation shows that in the GOE case

$$
N(A, \Delta \varepsilon)=\text { constant } \times \Delta \varepsilon A^{3} \exp \left(-\frac{A^{2}}{8 \sigma^{2}}\right)
$$

so that in this case

$$
P^{\prime}(A)=\frac{A^{2}}{2 \sqrt{\pi} \sigma^{3}} \exp \left(-\frac{A^{2}}{4 \sigma^{2}}\right)
$$

\section{Appendix 2}

The results of $\S 2.4$ assume that the occupation probabilities behave classically; if $P$ is the probability of a transition between levels $n, n+1$ when $X$ changes from $X_{0}$ to $X_{0}+\Delta X$, then the occupation probabilities transform as follows:

$$
\left(\begin{array}{c}
f_{n+1} \\
f_{n}
\end{array}\right) \rightarrow\left(\begin{array}{c}
f_{n+1}^{\prime} \\
f_{n}^{\prime}
\end{array}\right)=\left(\begin{array}{cc}
1-P & P \\
P & 1-P
\end{array}\right)\left(\begin{array}{c}
f_{n+1} \\
f_{n}
\end{array}\right) .
$$

This assumption is not justified in the case of a single avoided crossing, because of quantum interference effects: initially, at $X_{0}$, the two-level density matrix is given by

$$
\hat{e}=\left(\begin{array}{cc}
f_{n+1} & \chi \\
\bar{\chi} & f_{n}
\end{array}\right) \text {. }
$$


The transition from $X_{0}$ to $X_{0}+\Delta X$ is described by a unitary evolution operator

$$
\hat{U}=\left(\begin{array}{cc}
\alpha & \beta \\
-\bar{\beta} & \bar{\alpha}
\end{array}\right) \quad|\alpha|^{2}+|\beta|^{2}=1
$$

where $\alpha, \beta$ are complex numbers, and the transition probability is

$$
P=|\beta|^{2} \text {. }
$$

After the avoided crossing, at $X_{0}+\Delta X$, the final value of the density matrix is

$$
\hat{\rho}^{\prime}=\hat{U} \hat{\rho} \hat{U}^{+}=\left(\begin{array}{cc}
f_{n+1}^{\prime} & \chi^{\prime} \\
\chi^{\prime} & f_{n}^{\prime}
\end{array}\right)
$$

where

$$
f_{n+1}^{\prime}=(1-P) f_{n+1}+P f_{n}+\bar{\chi} \alpha \beta+\chi \bar{\alpha} \bar{\beta}
$$

etc. The final two terms in (A2.6) make this equation different from the classical result (A2.1).

When there are many avoided crossings between a large number of states, the expressions corresponding to (A2.6) will contain many of these quantum interference terms. In $\S 2.4$ it is assumed that these terms have random phases, so that they combine incoherently and can therefore be neglected.

\section{References}

Berry M V 1983 Chaotic Behaviour of Deterministic Systems (Les Houches Lectures XXXVI) ed G Iooss, R G H Helleman and R Stora (Amsterdam: North Holland) pp 172-271

Berry M V and Robnik M 1986 J. Phys. A: Math. Gen. 19 649-68

Bohigas O, Giannoni M J and Schmidt C 1984 Phys. Rev. Lett. 52 1-4

Büttiker M, Imry Y and Laundauer R 1983 Phys. Lett. 96A 356-9

Dyson F J 1962 J. Math. Phys. 3 157-65

Gefen Y and Thouless D J 1987 Phys. Rev. Lett. 59 1752-5

Greenwood D A 1957 Proc. Phys. Soc. 11 585-96

Hill D L and Wheeler J A 1952 Phys. Rev. $891102-45$

Kubo R 1956 Can. J. Phys. 34 1274-7

Mahan G D 1980 Many Particle Physics (New York: Plenum)

Porter C E (ed) 1965 Statistical Theory of Spectra: Fluctuations (New York: Academic)

Ott E 1979 Phys. Rev. Lett. 42 1628-31

Wilkinson M 1987 J. Phys. A: Math. Gen. 20 2415-23

1988 J. Phys. A: Math. Gen. 21 1173-90

Zener C 1932 Proc. R. Soc. A 137 696-702 